\title{
Molecular cloning and expression analysis of Aquaporin genes in pearl millet [Pennisetum glaucum (L) R. Br.] genotypes contrasting in their transpiration response to high vapour pressure deficits
}

\author{
Palakolanu Sudhakar Reddy ${ }^{\mathrm{a}}$, Murugesan Tharanya ${ }^{\mathrm{a}, \mathrm{b}}$, Kaliamoorthy Sivasakthi ${ }^{\mathrm{a}, \mathrm{b}}$, \\ Mallayee Srikanth $^{a}$, C. Tom Hash ${ }^{\mathrm{a}}$, Jana Kholova ${ }^{\mathrm{a}}$, Kiran K. Sharma ${ }^{\mathrm{a}}$, Vincent Vadez ${ }^{\mathrm{a}, *}$ \\ a International Crops Research Institute for the Semi-Arid Tropics (ICRISAT), Patancheru, Hyderabad 502 324, Telangana, India \\ b Department of Industrial Biotechnology, Bharathidasan University, Tiruchirappalli, India
}

\section{A R T I C L E I N F O}

\section{Keywords:}

Aquaporins

Pearl millet

Transpiration rate

Vapour pressure deficit

VPD-sensitive genotype

VPD-insensitive genotype

\begin{abstract}
A B S T R A C T
Pearl millet is a crop of the semi-arid tropics having high degree of genetic diversity and variable tolerance to drought stress. To investigate drought tolerance mechanism that possibly accounts for differences in drought tolerance, four recombinant inbred lines from a high resolution cross (HRC) were selected for variability in their transpiration rate ( $\mathrm{Tr}$ ) response to vapour pressure deficit (VPD) conditions. The differential Tr response of the genotypes to increased VPD conditions was used to classify the genotypes as sensitive or insensitive to high VPD. Aquaporin (AQP) genes PgPIP1;1, PgPIP1;2, PgPIP2;1, PgPIP2;3, PgPIP2;6, PgTIP1;1 and PgTIP2;2 were cloned. Phylogenetic analysis revealed that the cloned PgAQPs were evolutionarily closer to maize AQPs than to rice. $P g A Q P$ genes, including PgPIP1;1 and PgPIP2;6 in root tissue showed a significant expression pattern with higher expression in VPD-insensitive genotypes than VPD-sensitive genotypes under low VPD conditions (1.2 kPa) i.e when there is no high evaporative demand from the atmosphere. PgAQP genes (PgPIP2;1 in leaf and root tissues; PgPIP1;2 and PgTIP2;2 in leaf and PgPIP2;6 in root) followed a diurnal rhythm in leaves and roots that have either higher or lower expression levels at different time intervals. Under high VPD conditions (4.21 kPa), PgPIP2;3 showed higher transcript abundance in VPD-insensitive genotypes, and PgPIP2; 1 in VPD-sensitive genotypes, while rest of the PgAQPs showed differential expression. Our current hypothesis is that these differences in the expression of $A Q P$ genes under different VPDs suggests a role of the AQPs in tuning the water transport pathways with variation between genotypes.
\end{abstract}

\section{Introduction}

Pearl millet [Pennisetum glaucum (L.) R. Br.], an annual diploid crop belonging to the Poaceae family, is the sixth most important global cereal crop [1]. It is a highly drought tolerant crop that can grow on marginal lands, under high temperatures $>40{ }^{\circ} \mathrm{C}$ [2] and low rainfall (200-600 mm) conditions [3]. Despite the hardiness of pearl millet, there is ample genetic variation for drought adaptation within the species which can be used to develop climate change-ready improved cultivars. Drought stress is a major abiotic stress factor limiting yield and yield stability of crops which may become even more severe in future climatic scenarios. Major research has been focused towards understanding drought tolerance in major crops like soybean, wheat and rice [4-6], but less in pearl millet. Recent studies indicate that water saving traits would explain the terminal drought adaptation of pearl millet cultivars under some of the drought scenarios [7-11].
Therefore, to harness these traits towards crop improvement, a better understanding of the mechanisms involved in water saving, and their role in key drought adaptive phenotypes is needed.

Part of these water saving traits lay in the capacity of certain genotypes to restrict plant transpiration when the evaporative demand is high [12]. The physiological basis of drought adaptive phenotypes has been partially elucidated in pearl millet genotypes carrying a terminal drought tolerance quantitative trait locus (QTL). The QTL carriers had lower transpiration rate (Tr) compared to sensitive lines and were able to restrict their $\operatorname{Tr}$ further upon rising vapor pressure deficit (VPD) under well-watered conditions [8]. This contributed to water saving at a vegetative stage, making more water available for reproduction and grain filling [12]. This trait was also hypothesized to be explained by hydraulic limitations in the soil-plant atmosphere continuum [13]. Water uptake by the roots and loss from the leaves are indeed driven by hydrostatic and osmotic forces to keep the plants in a proper water

\footnotetext{
* Corresponding author at: Physiology Laboratory, International Crops Research Institute for the Semi-Arid Tropics (ICRISAT), Patancheru, Hyderabad 502 324, Telangana, India.

E-mail address: v.vadez@cgiar.org (V. Vadez).
} 
balance by continuously adjusting the water conductance in tissues, especially roots and leaves. The movement of water through the root cylinder occurs through three parallel paths: across cell walls (apoplastic), from cell to cell across or the plasmodesmata (symplastic), or traversing the cell membranes (transcellular) [17], although, the symplastic and transcellular pathways cannot be easily distinguished. Both depend on the function of Aquaporins (AQPs) to carry water through membranes. AQPs are specialized membrane transport proteins that play a crucial role in water transport in many plant organs, including the roots $[18,19]$. Since the AQPs are being considered as facilitators of water movement, we hypothesized that the expression of AQPs would increase under high evaporative demands, more so in lines that show no transpiration restriction when compared to the lines that show transpiration restriction under high VPD. Based on the degree to which their transpiration was affected upon AQP inhibitor treatments, genotypes contrasting for transpiration response under high VPD indeed varied for the utilization of water transport pathways (Tharanya et al., Unpublished). Similarly in soybean, the transpiration rate response under high VPD was found to be contrasting [14], where the decline in transpiration upon inhibition of aquaporins (AQPs) varied between VPD-sensitive and -insensitive genotypes $[15,16]$. Therefore, it is important to study the role of AQPs at molecular level to investigate a possible relationship to a critical physiological response, i.e., in the capacity to restrict transpiration under high VPD.

Aquaporins are small integral membrane water channeling proteins that belong to the ancient family of major intrinsic proteins (MIPs) and have been found in most living organisms, primarily in facilitating water transport [20-22]. AQPs are widely involved during different stages of plant development [23-27] and in response to diverse abiotic stresses [27-31]. Plant AQPs have distinct AQP isoforms that are targeted to their specific functions in response to different environmental conditions showing a high multiplicity of isoforms with 35, 33, 41, 41, 47 and 33 homologs in Arabidopsis, maize, sorghum, potato, tomato and rice, respectively [21,27,32-35]. As such, there is so far no aquaporin gene reported in pearl millet and part of this study consisted in cloning pearl millet aquaporin genes using an homology-based cloning approach. This study was also about assessing the relationship between pearl millet homologs and those in other species.

The present study is therefore aimed at: (i) cloning pearl millet $A Q P$ genes using homology based gene cloning approach, (ii) carry out a molecular and physiological characterization of these genes; (iii) assess a possible link between transpiration rate response to high VPD in pearl millet genotypes contrasting for that phenotype and the expression of $A Q P$ genes in root and leaf tissues collected under different atmospheric VPD conditions and times of the day.

\section{Materials and methods}

\subsection{Plant material and growth conditions}

The transpiration rate ( $\mathrm{Tr}$, in $\mathrm{mg} \mathrm{cm}{ }^{-2} \mathrm{~min}^{-1}$ ) was assessed in two pairs of pearl millet genotypes that were initially identified as contrasting for the transpiration response to increasing VPD. These lines belonged to a high resolution cross (HRC) earlier developed by crossing one of the most promising near isogenic line, ICMR 01029, containing a terminal drought tolerance QTL, to a drought sensitive line H77/833-2 $[12,36]$. ICMR 01029 is a $\mathrm{BC}_{4} \mathrm{~F}_{3}$ introgression of a terminal drought tolerance QTL from tolerant donor PRLT-2/89-33 into drought sensitive H77/833-2 (See [8] for details). HRC contrasting genotypes were therefore VPD-insensitive (ICMR 1122 and ICMR 1086) and VPD-sensitive (ICMR 1152 and ICMR 1078). Plants were grown in 8" plastic pots filled with $5.5 \mathrm{~kg}$ of sand collected from the ICRISAT farm and saturated with nutrient solution. Each pot was sown with eight seeds and thinned to two healthy seedlings after a week and grown under well-watered conditions up to 28 days. Ten most uniform plants of each genotype were selected from 10 replica pots of each genotype for
Table 1

List of the primers used for homology based cloning and qPCR. DF - Degenerate Forward; DR - Degenerate Reverse; qF - qPCR Forward; qR - qPCR Reverse.

\begin{tabular}{|c|c|c|}
\hline S. No. & Gene & Sequence $\left(5-3^{\prime}\right)$ \\
\hline 1 & PgPIP1;1 & $\begin{array}{l}\text { DF: ATGGAGGGGAARGAGGAGGACGTGCG } \\
\text { DR: TTAAGACCTGCTCTTGAAYGGGATSGCYCT } \\
\text { qF: ACAGGGATCACGCCTGGAGC } \\
\text { qR: AGTCGCGGCTCTTGAAGG }\end{array}$ \\
\hline 2 & PgPIP1;2 & $\begin{array}{l}\text { DF: ATGGAGGGSAARGAGGAGGAYGTNCGSCTGG } \\
\text { DR: TTANGACCTGCTCTTGAAYGGRATNGCNCT } \\
\text { qF: AGGAGCCAAGCCTGGGAT } \\
\text { qR: AGACCTGCTCTTGAATGGGATG }\end{array}$ \\
\hline 3 & PgPIP2;1 & $\begin{array}{l}\text { DF: ATGGGSAAGGACGASGTGATSGAGAGCG } \\
\text { DR: TCAYGCGTTGCTCCTGAAGGAGCCGAGGGC } \\
\text { qF: AACAAGGACAAGCCATGGGAT } \\
\text { qR:AGGGCCTTGATGGCGCCGGCC }\end{array}$ \\
\hline 4 & PgPIP2;3 & $\begin{array}{l}\text { DF: ATGGCSAARGACATYGAGGCSGCGGCGGC } \\
\text { DR: CTAGCSGCKGAAGGAGGMNGANGAKCCSAGCTT } \\
\text { qF: CGCGGTTTTCATGGTCCACC } \\
\text { qR: TCCCAGGCCTTGTCGTTGTT }\end{array}$ \\
\hline 5 & PgPIP2;6 & $\begin{array}{l}\text { DF: ATGGGYAARGAGGTSGAYGTGTCCRCTCT } \\
\text { DR: CTASGCGTTGCTCCGGAARGAGCCGTA } \\
\text { qF: AATAACAGCAAGGCCTGGAGC } \\
\text { qR: AGCCGTAGCCCCTGGCGCTGGC }\end{array}$ \\
\hline 6 & PgTIP1;1 & $\begin{array}{l}\text { DF: ATGCCGATCMRNARSATCGCCSTSGGGAGC } \\
\text { DR: TTAGTAGTCGGTGGWGGGKAGCTGCTCGTGGGT } \\
\text { qF: TGGTCCTGGGGGTACCAG } \\
\text { qR: GCTGCTCGTGGGTGTGGGAG }\end{array}$ \\
\hline 7 & PgTIP2;2 & $\begin{array}{l}\text { DF: ATGTCGGGCAACATCGCCTTCGGCCGCT } \\
\text { DR: TTAGAASTCGCTGCTGGCRACGGGGGC } \\
\text { qF: CGGCGACTTTACCAACATC } \\
\text { qR: CGGGGGCGTGGTCTCCGCAC }\end{array}$ \\
\hline 8 & $P g M D H$ & $\begin{array}{l}\text { qF: AGAAGGCGCTTGCTTACTCAT } \\
\text { qR: CAGTTCTGGGTGAGGGAATCT }\end{array}$ \\
\hline 9 & $P g U B C$ & $\begin{array}{l}\text { qF: TTCAAACCTCCGAAGGTGTCTT } \\
\text { qR: GGCTCCACTGCTCTTTAAGAATG }\end{array}$ \\
\hline
\end{tabular}

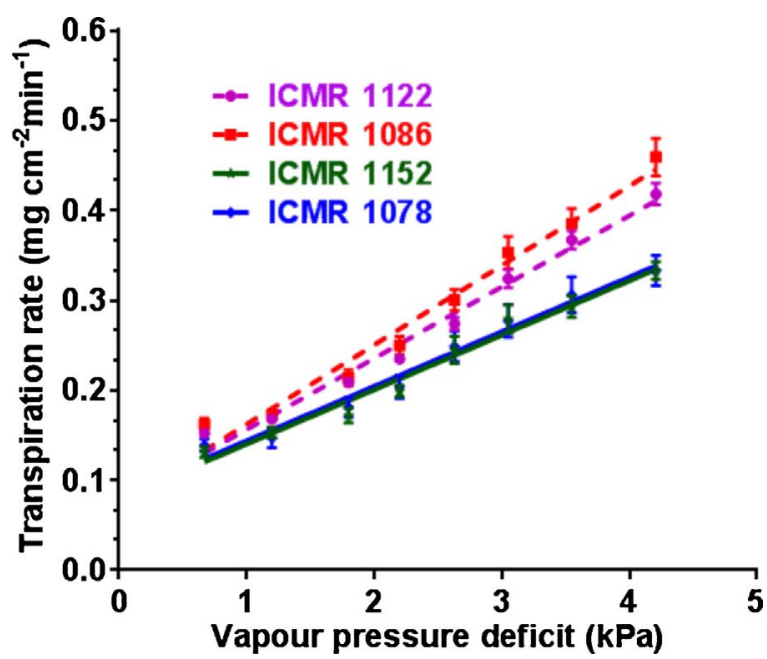

Fig. 1. Tr response to increasing VPD in High Resolution Cross (HRC) lines showing genotypes with dashed lines (ICMR 1122 and ICMR 1086) and solid lines (ICMR 1152 and ICMR 1078)

experiments in the controlled growth chambers. A thermo-hygrograph sensor (Tinytag Ultra 2 TGU-4500 Gemini Data loggers Ltd, Chichester, UK) was positioned within the plant canopies in the growth chamber for regular record of the air temperature and relative humidity during the measurement period. On the day before the experiment, the pots were well-watered, allowed to drain to reach field capacity and bagged with a transparent plastic bag wrapped around the plant stem to 
Table 2

Regression results for the transpiration rate ( $\mathrm{Tr})$ in response to increasing VPD were assessed in four pearl millet genotypes [ICMR 1152 and ICMR 1078 (VPD-sensitive \& low Tr), ICMR 1122 and ICMR 1086 (VPD-insensitive \& high Tr)] contrasting for VPD response.

\begin{tabular}{llllll}
\hline Genotypes & $\begin{array}{l}\text { Mean Tr } \\
\left(\mathrm{mg} \mathrm{cm}^{-2} \mathrm{~min}^{-1}\right)\end{array}$ & $\begin{array}{l}\text { Break } \\
\text { point }\end{array}$ & Slope & SE & $\mathrm{R}^{2}$ \\
\hline ICMR 1152 & 0.210 & No & $0.0605 \pm 0.00376$ & 0.009 & 0.977 \\
ICMR 1078 & 0.214 & No & $0.0606 \pm 0.00367$ & 0.010 & 0.978 \\
ICMR 1122 & 0.247 & No & $0.0793 \pm 0.0044$ & 0.006 & 0.981 \\
ICMR 1086 & 0.262 & No & $0.0882 \pm 0.0056$ & 0.005 & 0.976 \\
\hline
\end{tabular}

prevent soil water evaporation during the evaluation of plant transpiration. Five replications of each genotype were placed in two different chambers individually. On the following day, one chamber was maintained at $1.2 \mathrm{kPa}$ throughout the experiment (hereafter referred as low VPD chamber), while the other chamber had a ladder of VPD ranging from $0.69 \mathrm{kPa}$ to $4.21 \mathrm{kPa}$ (hereafter referred as high VPD chamber). In the high VPD chamber, each VPD was maintained for about $45 \mathrm{~min}$ and in between successive VPD steps, 15 min transition was allowed for the plants to adjust and stabilize the conditions in the growth chamber. The low VPD chamber allowed the comparison of gene expression between low and high VPD in the afternoon. The light was turned on at 6:00 am. in the morning and the beginning of the VPD ladder that started at 7:45 am.

\subsection{Treatments and sample collection}

The plants were grown in glasshouse in pots filled with sand (to facilitate root tissue collection) and routinely irrigated with nutrient solution on alternate days. A day before experiment, the plants were moved to growth chamber during late afternoon for night acclimatization. The plants were acclimatized to day regime VPD of $1.8 \mathrm{kPa}$ (3:00 to $18: 30$ ) and night regime (19:30 to 5:30 h) VPD of $0.67 \mathrm{kPa}$. Between 18:30 to $19: 30 \mathrm{~h}$ there was a transition period for night regime. From 5:30 to $6: 30 \mathrm{~h}$ again, there was a transition period for day regime. The following morning $(8: 00 \mathrm{~h})$ on the day of experiment, the leaf and root samples were collected at $1.2 \mathrm{kPa}$ (3 plants per genotype). Thereafter, the VPD was increased progressively in the treatment chamber (typically covering a range of $0.69-4.21 \mathrm{kPa}$ ), whereas the control chamber was kept constantly under low VPD (1.2 kPa) condition. At each VPD, the plants were weighed gravimetrically with $0.01 \mathrm{~g}$ precision scale (PE 12, Mettler Toledo, Schweiz-GmbH, Germany) to measure the transpiration values. At the end of the VPD ladder at $4.21 \mathrm{kPa}$ at 15:00 pm, leaf and root samples were again collected from both the control $(1.2 \mathrm{kPa})$ and the treatment chamber $(4.21 \mathrm{kPa})$ and frozen immediately in liquid nitrogen and stored in $-80^{\circ} \mathrm{C}$ for RNA isolation. Further, leaf area was measured using leaf area meter (LI3100, Li-Cor, Lincoln, Nebraska, USA) and transpiration rate (Tr), i.e., water loss per unit of leaf area was calculated. The breakpoint response of transpiration to increasing VPD in the growth chamber was analyzed with segmental regression analysis using Graph Pad Prism version 6
(GraphPad software, Inc., CA, and USA) which provides $\mathrm{r}^{2}$ and slope value.

\subsection{Total RNA isolation}

Total RNA was isolated from pearl millet plants exposed to low and high VPD by NucleoSpin RNA plant kit (Macherey-Nagel, Duren, Germany) following the recommended procedures including on column DNase I treatment. The concentration and purity of RNA was determined using NanoVue plus spectrophotometer (GE health care, USA) and BioAnalyzer (Agilent) to ascertain the ratio of the absorbance at 260 and $280 \mathrm{~nm}(260 / 280)$ of the samples that ranged from 1.8 to 2.0, and the integrity of RNA verified by agarose gel electrophoresis $(1.4 \%$ agarose). The obtained RIN values for all the samples were $>8$. Total RNA isolated from the control and treated samples were diluted to $100 \mathrm{ng} / \mu \mathrm{l}$ concentrations for use in quantitative real-time PCR (qPCR).

\section{4. cDNA synthesis and cloning of PgAQP genes}

Two micrograms of total RNA was used to synthesize the complimentary DNA (cDNA) by using the SuperScript III first strand synthesis kit (Invitrogen, USA). This cDNA was used as a template for the cloning of $A Q P$ genes using respective degenerate gene-specific forward and reverse primers. Degenerate primers were designed based on conserved sequences found in the closely related monocot species like rice, sorghum and maize (Table 1). The cDNA sequences of PgAQPs were PCR amplified using $150 \mathrm{ng}$ of each primer along with $200 \mu \mathrm{M}$ of dNTPs, 2.5 units of Taq DNA polymerase (Invitrogen) and $1 \mu \mathrm{l}$ of cDNA as template in a $50 \mu \mathrm{l}$ reaction. PCR reaction conditions were $94^{\circ} \mathrm{C}, 1 \mathrm{~min} ; 55^{\circ} \mathrm{C}$, $1 \mathrm{~min}$ and $72{ }^{\circ} \mathrm{C}, 1 \mathrm{~min}$ for 30 cycles, followed by a final extension at $72{ }^{\circ} \mathrm{C}$ for $10 \mathrm{~min}$. The PCR amplicons were verified through agarose gel electrophoresis and cloned into pCR4.0 TOPO TA vector (Invitrogen) according to the manufacturer's protocol and sequenced completely.

\subsection{Sequence analysis}

The nucleotide sequence information generated from the cloned $P g A Q P$ genes were annotated by using BlastN and BlastX of NCBI databases to search for sequence similarity between processed DNA-sequence outputs and public non-redundant nucleotide and amino acid databases. Classification and nomenclature of $P g A Q P$ genes was done according through a phylogenetic tree that was generated from pearl millet, rice and maize translated AQP cDNA sequences using MEGA6. Conserved motifs of cloned PgAQP proteins were detected using Multiple Em for Motif Elicitation (MEME) software (http://meme.nbcr. net). The aligned sequences were used to find the conserved domains, forger positions and ar/R motifs present in the sequences of PgAQP aligned sequences. Transmembrane helices and protein subcellular localization of PgAQP proteins were performed using the TMPRED (http://www.ch.embnet.org/software/TMPRED_form.html) and CELLO v.2.5 (http://cello.life.nctu.edu.tw/) programmes, respectively. In silico sequence analysis like number of amino acids (AA), molecular weight

Table 3

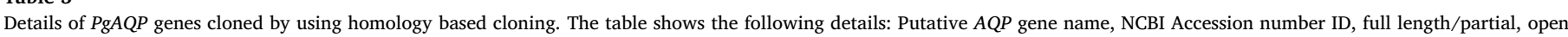

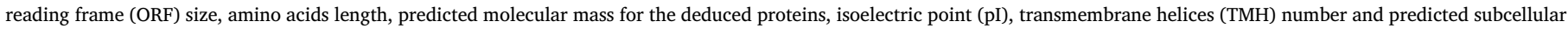
localization (SL).

\begin{tabular}{|c|c|c|c|c|c|c|c|c|c|}
\hline S. No. & Gene & Full/Partial & GenBank Acc. & ORF (bp) & Protein (aa) & Protein size $(\mathrm{kDa})$ & pI & TMH & SL \\
\hline 1 & PgPIP1;1 & Full & KU532909 & 867 & 288 & 30.74 & 9.84 & 6 & Plasma membrane \\
\hline 2 & PgPIP $1 ; 2$ & Full & KU532910 & 867 & 288 & 30.70 & 9.53 & 6 & Plasma membrane \\
\hline 3 & PgPIP2;1 & Full & KU532911 & 873 & 290 & 30.32 & 8.99 & 6 & Plasma membrane \\
\hline 4 & PgPIP2;3 & Partial & KU532912 & 636 & 212 & 22.06 & 10.81 & 4 & Plasma membrane \\
\hline 5 & PgPIP2;6 & Full & KU532913 & 861 & 286 & 29.91 & 9.34 & 7 & Plasma membrane \\
\hline 6 & PgTIP1;1 & Full & KU532914 & 750 & 249 & 25.73 & 6.71 & 6 & Plasma membrane \\
\hline 7 & PgTIP2;2 & Full & KU532915 & 747 & 248 & 25.14 & 7.18 & 6 & Plasma Membrane \\
\hline
\end{tabular}




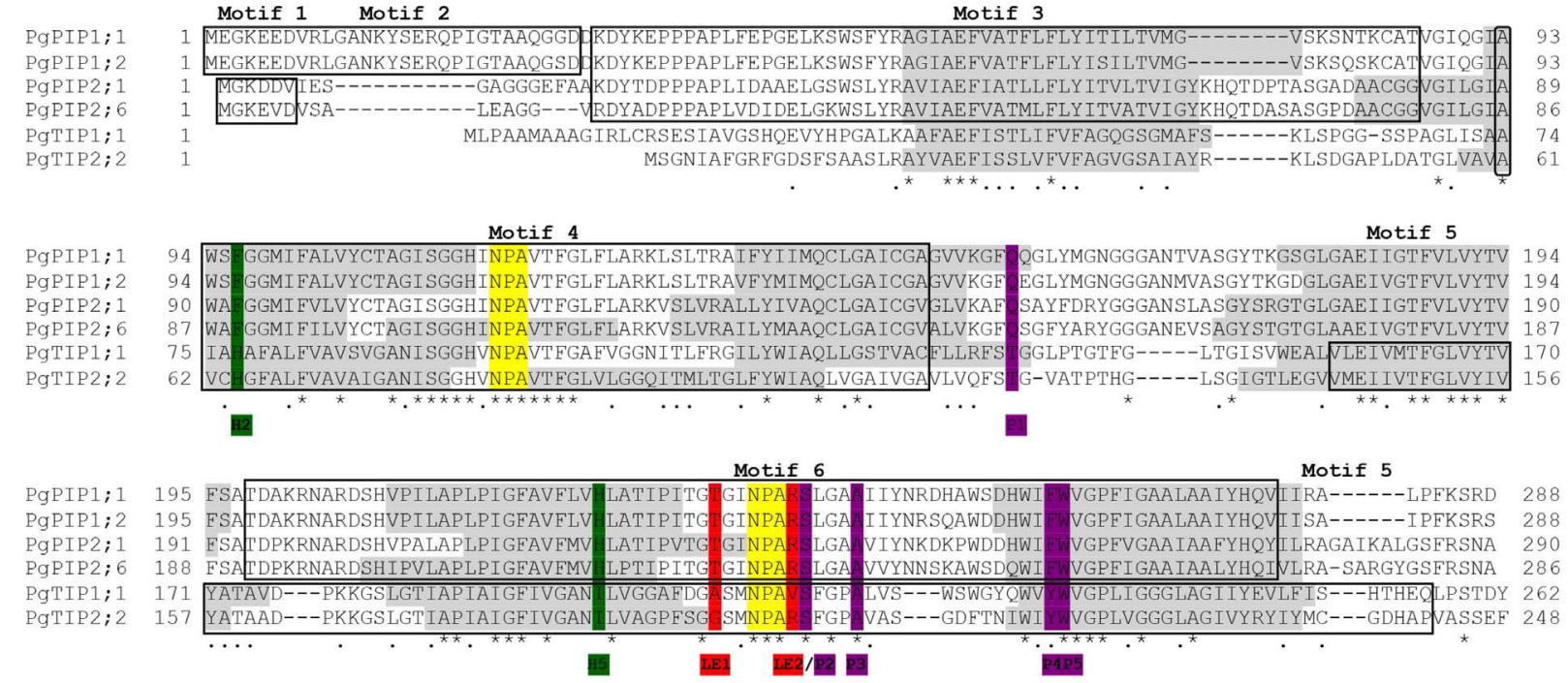

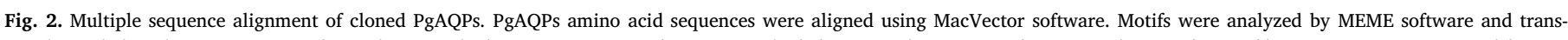

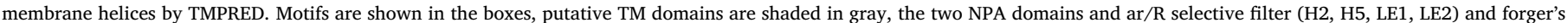

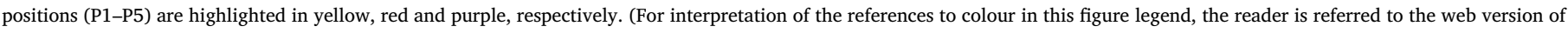
this article.)

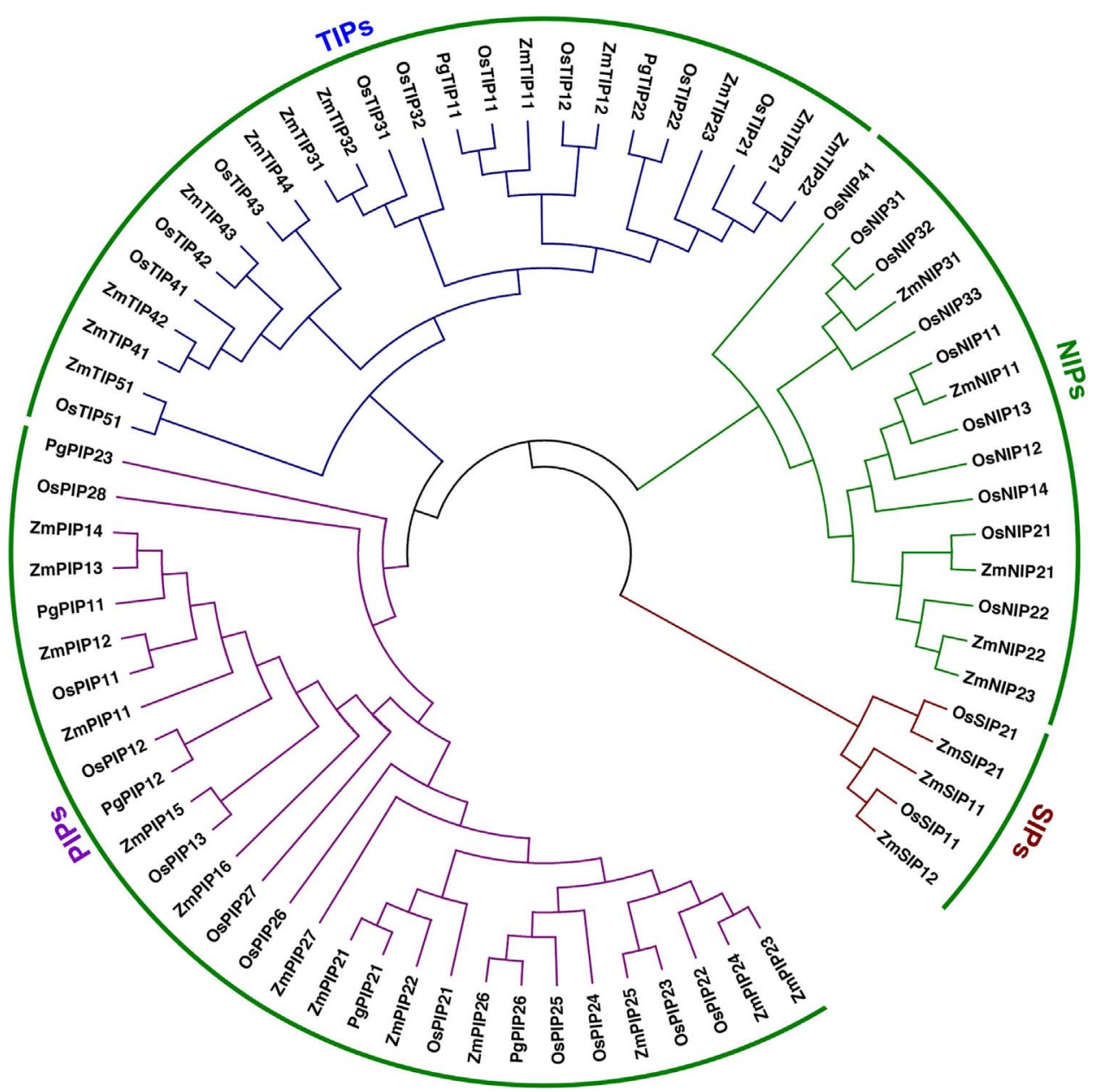

Fig. 3. Phylogenetic relationship of PgAQP proteins with maize and rice AQP proteins. The neighborjoining tree was constructed for all PgAQP protein sequences and their respective homologs from maize and rice. The AQPs fall in to four clades, viz NIPs, TIPs, SIPs and PIPs, with PgPIPs showing a close relation with PIPs from Zea mays.

(MW) and theoretical isoelectric point (pI) of the PgAQP proteins were predicted by using MacVector software (V14.5.0).

\subsection{Quantitative real-time PCR ( $q P C R)$}

qPCR reactions were performed in optical 96-well plates on a Realplex (Eppendorf, Germany). Reactions were performed in a total volume of $10 \mu$ containing $1 \mu$ l of total RNA (100 ng), $400 \mathrm{nM}$ of each 

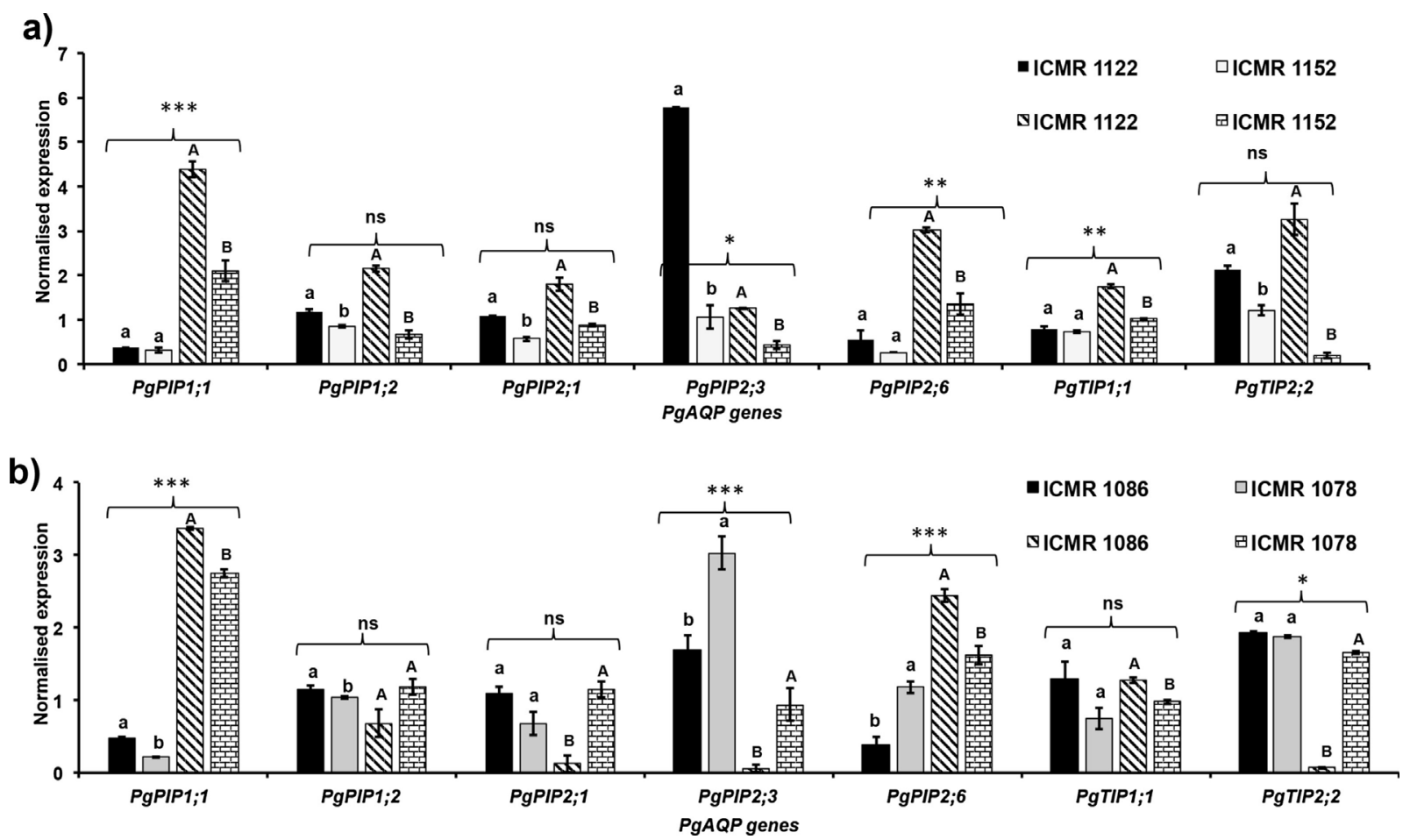

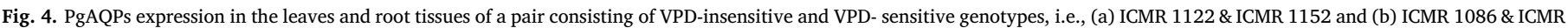

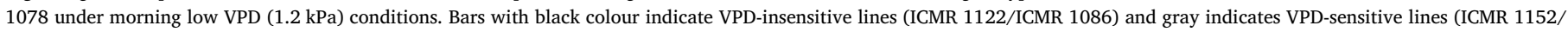

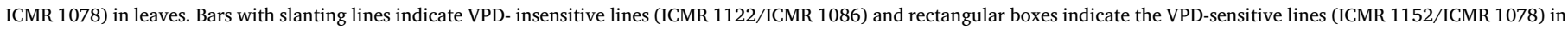

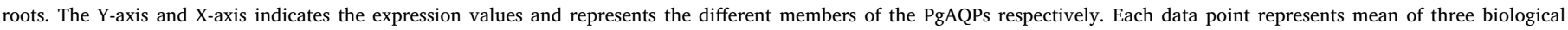

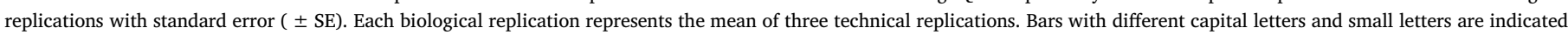

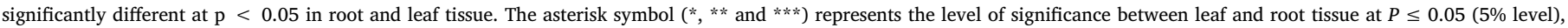
$P \leq 0.01$ (1\%level) and $P \leq 0.005$ (0.5\% level). "ns" indicates the non-significant values.

primer, $5 \mu \mathrm{l}$ of $2 \mathrm{X}$ one step SYBR RT-PCR buffer 4 (Takara, Japan) and $0.5 \mu l$ of Prime Script One Step Enzyme Mix 2 (Takara, Japan), and the reaction volume made up to $10 \mu \mathrm{l}$ with RNase-free $\mathrm{H}_{2} \mathrm{O}$. qPCR reactions of all samples were done by following standard thermal profile: $42{ }^{\circ} \mathrm{C}$ for $5 \mathrm{~min}$ and $95^{\circ} \mathrm{C}$ for $10 \mathrm{~s}$ (reverse transcription) followed by $40 \mathrm{cy}-$ cles of $15 \mathrm{~s}$ at $95^{\circ} \mathrm{C}, 15 \mathrm{~s}$ at $62^{\circ} \mathrm{C}$ with fluorescent signal recording and $15 \mathrm{~s}$ at $72{ }^{\circ} \mathrm{C}$. Amplicon dissociation curves were recorded after $40 \mathrm{cy}-$ cles by heating from 58 to $95{ }^{\circ} \mathrm{C}$ with fluorescence measured within $20 \mathrm{~min}$. The experiments were independently repeated three times and the data from these experiments were averaged. The relative fold change in expression levels of PgAQP transcripts in response to different VPD treatments was estimated using qbase + software [37] by normalizing with Malate dehydrogenase (MDH) and Ubiquitin-conjugating enzyme (UBC) reference genes [38].

\subsection{Data analysis}

Transpiration response to increasing VPD ( $0.7 \mathrm{kPa}$ to $4.21 \mathrm{kPa})$ was analyzed with the linear regression in Graph pad prism version 6 (graph pad software. Inc, CA. and USA) which provides a breakpoint, slope and $\mathrm{r}^{2}$ value. Normalized expressions of AQPs in different time points were normalized against $P g M D H$ and $P g U B C$ reference genes using qbase + software (ver: 2.4; Biogazelle, Belgium) [37]. The relative expression of $P g A Q P$ genes under high VPD (4.21 kPa) conditions was analyzed using qbase + software [37], where the data was normalized against two reference genes (PgMDH and $P g U B C$ ) and low VPD in leaf and root samples collected at the same time of the day (afternoon). Statistical analysis of $A Q P$ gene expression between VPD-insensitive and VPDsensitive genotypes under different VPD conditions (low and high VPD) and tissues (root and leaf) were analyzed with statistical program package CoStat version 6.204 (Cohort Software, Monterey, CA, USA). One-way ANOVA was carried out to test for genotypic difference between the VPD-insensitive and VPD-sensitive genotypes. Means were compared using Tukey-Kramer test and LSD at $P \leq 0.05$ (5\% level), $P \leq 0.01$ (1\%level), $P \leq 0.005$ (0.5\% level).

\section{Results}

\subsection{Transpiration response to progressive VPD conditions}

The transpiration rate ( $\mathrm{Tr}$ ) in response to increasing VPD was tested in VPD-insensitive (ICMR 1122 and ICMR 1086) and VPD-sensitive (ICMR 1152 and ICMR 1078) genotypes of pearl millet. Upon increasing VPD, Tr increased in all the tested genotypes, although the increasing trend clearly varied with the genotype (Fig. 1). In all the four tested genotypes, Tr increased linearly with rising VPD and there was no breakpoint at any VPD level throughout the tested VPDs. Therefore, Tr data could be fitted to a single linear curve with a good fit in all genotypes $\left(\mathrm{r}^{2}>0.90\right)$ (Fig. 1, Table 2). There were indeed significant differences among the genotypes in the values of slope characterizing the response to $\operatorname{Tr}$ to increasing VPD. VPD-sensitive ICMR 1152 and ICMR 1078 had a lower $\mathrm{Tr}$ slope $\left(0.0605\right.$ and $\left.0.0606 \mathrm{mg} \mathrm{cm}^{-2} \mathrm{~min}^{-1}\right)$ than VPD-insensitive ICMR 1122 and ICMR 1086 (0.0793and $0.0882 \mathrm{mg} \mathrm{cm}^{-2} \mathrm{~min}^{-1}$ ) under high VPD conditions (Fig. 1, Table 2).

\subsection{Cloning and sequence analysis of $A Q P$ genes in pearl millet}

Seven $A Q P$ genes were cloned from pearl millet using homologybased cloning approach which include five plasma membrane aquaporins [PIPs] (PgPIP1;1, PgPIP1;2, PgPIP2;1, PgPIP2;3 and PgPIP2;6) and two tonoplast intrinsic aquaporins [TIPs] (PgTIP1;1 and PgTIP2;2). The cloned PgAQP sequences were deposited in the GenBank (Table 3). Sequence analysis of the cloned PgAQP protein sequences showed less sequence variation in length of amino acids (248 [PgTIP2;2] to 290 

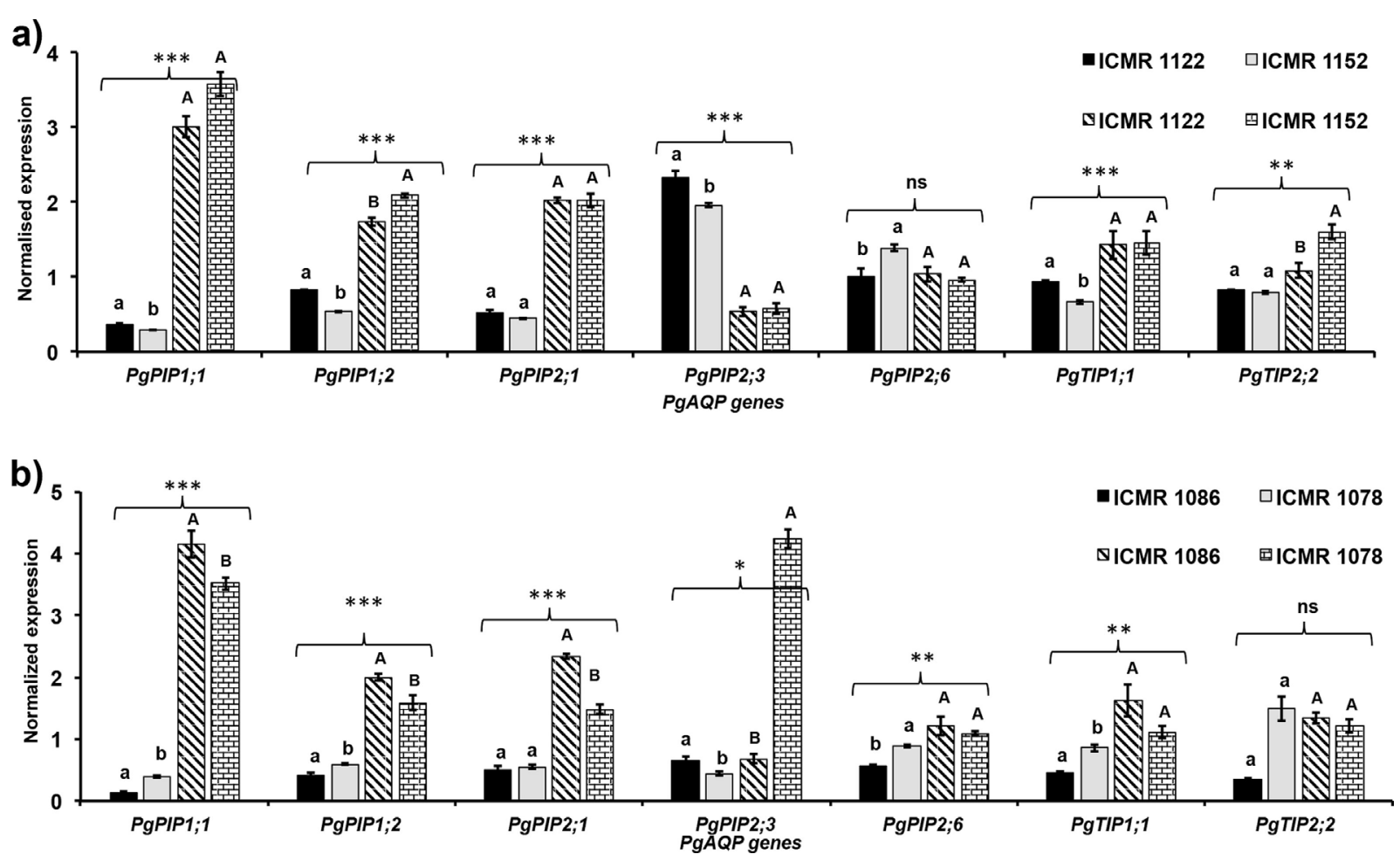

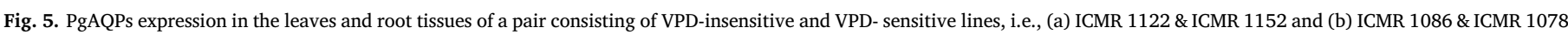

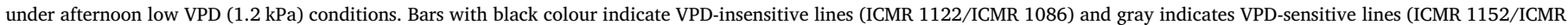

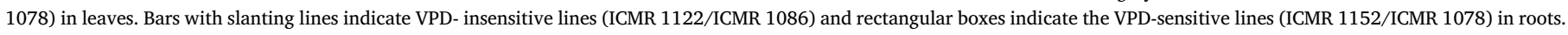

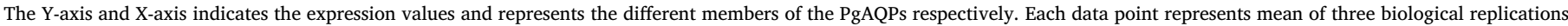

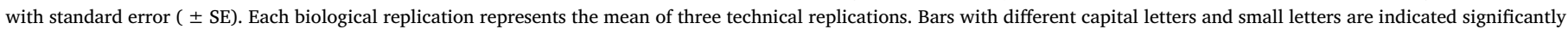

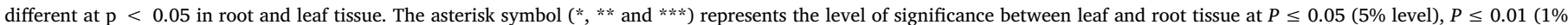
level) and $P \leq 0.005$ ( $0.5 \%$ level). "ns" indicates the non-significant values.

[PgPIP2;1]), molecular weight (ranging from 25.14 [PgTIP2;2] to $30.74 \mathrm{kDa}$ [PgPIP1;1]) and high variability in isoelectric point (pI) values (ranging from 6.71 [PgTIP1;1] to 9.84 [PgPIP1;1]). The subcellular localization prediction revealed that all cloned PgAQP proteins belonged to plasma membrane (Table 3). In silico predictions showed that the number of transmembrane helices ranged from 6 to 7 ; PgPIP2;6 protein had the highest number with seven transmembrane helices (Table 3). The MEME motif scan analysis revealed that PgAQP gene family contained six conserved motifs with different sizes (Fig. 2 and Fig. S1). Multiple sequence alignment of PgAQP proteins shows that the sequences for NPA motif, ar/R selectivity filter and forger positions were highly conserved within the subfamily. Dual NPA motif and forger position (P1-P5) are highly conserved, but few did not match with those particular motifs were diverse between the subfamily (H2, H5, LE1, LE2) (Fig. 2). Further more, some deviations between the subfamilies were also observed (Fig. 2) indicating possible structural differences in different AQP subfamilies of pearl millet.

\subsection{Phylogenetic tree analysis}

PgAQP proteins together with Zea mays and Oryza sativa AQPs were used for finding their evolutionary relationships by constructing a phylogenetic tree using MEGA6 by neighbor joining method. All PgAQPs could be grouped into the same sub-clusters along with $Z$. mays and $O$. sativa AQPs, which indicated that they were conserved across these plant species (Fig. 3). The cloned PgAQPs were named and classified in a systematic manner based on their phylogenetic relationship with Z. mays and O. sativa AQPs (Fig. 3) [21,33]. Out of seven PgAQPs, five belonged to the PIP subfamily (PgPIP1;1, PgPIP1;2, PgPIP2;1, PgPIP2;3 and PgPIP2;6) and remaining two belonged to the TIP subfamily (PgTIP1;1 and PgTIP2;2). Cloned PgAQP family members had a wide range of sequence similarity from 61\% (PgPIP2;3) - 98\% (PgPIP2;1) with corresponding AQP family members from $Z$. mays and O. sativa (Figs. 2 and 3 ).

\subsection{VPD, tissue-specific and genotype-dependent expression of PgAQP genes}

Pearl millet genotypes showing differential Tr response to different VPD conditions were used to quantify the $P g A Q P$ gene expression levels in leaf and root tissues at different time points by qPCR. Most of the $P g A Q P$ genes showed higher expression in roots as compared to the leaves in the morning and afternoon, under low VPD conditions (1.2 kPa) in both VPD-insensitive (ICMR 1122 and ICMR 1086) and VPD-sensitive genotypes (ICMR 1152 and ICMR 1078) with few exceptions (Figs. 4-6). PgAQP genes, including PgPIP1;1, PgPIP1;2, PgPIP2;1 and PgTIP1;1 showed higher expression in the root tissue during afternoon (Fig. 5). In ICMR 1086 and ICMR 1078, the expression of PgPIP2;3 and PgTIP2;2 showed a higher expression in the leaf tissue compared to roots during morning (Fig. 4b). PgAQP genes, including PgPIP1;1 and PgPIP2;6 in root, showed higher expression during morning in VPD-insensitive genotypes (ICMR1122 and ICMR 1086) than VPD-insensitive genotypes (ICMR 1122 and ICMR 1152) (Fig. 4a and b). PgPIP2;3 and PgTIP2;2 in leaf showed higher expression in VPDinsensitive genotypes (ICMR 1122) than VPD-insensitive genotype (ICMR 1152) during morning hours (Fig. 4a). Interestingly, PgAQP genes (PgPIP2;1 in leaf and root tissues (Fig. 7a and b); PgPIP1;2 and PgTIP2;2 in leaf (Fig. 7a) and PgPIP2;6 in root (Fig. 7b) followed a diurnal rhythm in leaves and roots that had either higher or lower expression levels at different time intervals (Fig. 7). Under high VPD conditions when normalized with reference genes, PgAQP genes expression levels were higher in roots than leaves in both VPD-sensitive 

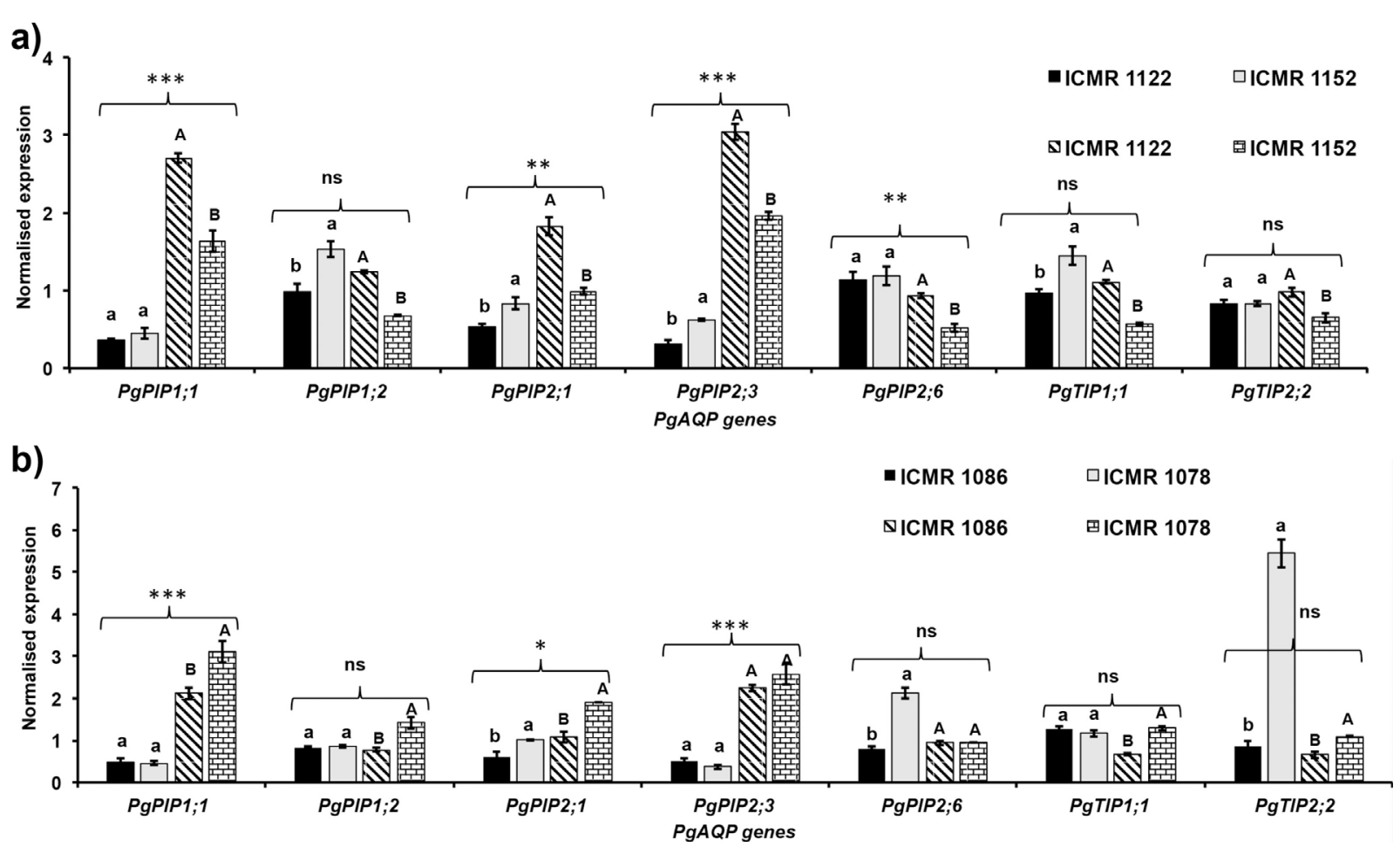

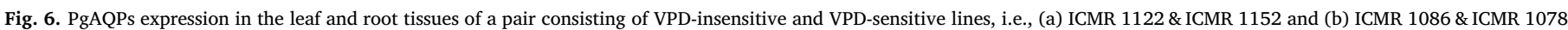

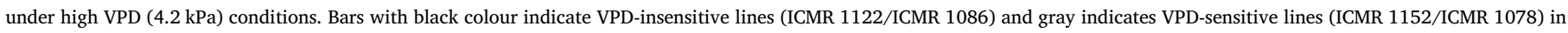

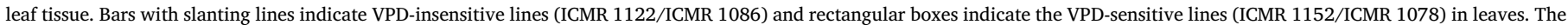

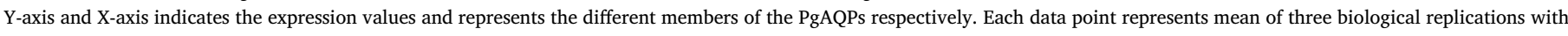

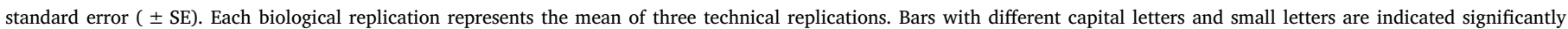

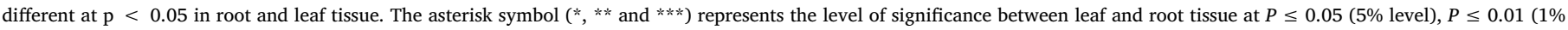
level) and $P \leq 0.005$ (0.5\% level). "ns" indicates the non-significant values.

and -insensitive lines with some exceptions (PgPIP1;2 in ICMR 1122 and ICMR 1078; PgTIP2;2 in ICMR 1086; ICMR 1078 and ICMR 1152, PgPIP2;6 in ICMR 1122, ICMR 1152 and in ICMR 1078, PgTIP1;1 in ICMR 1086 and ICMR 1152) (Fig. 6). Clearly, PgPIP1;1 and PgPIP2:3 showed higher expression in roots of the VPD-insensitive genotype (ICMR 1122) than VPD-sensitive genotype (ICMR 1152) (Fig. 6a). Overall, $P g A Q P$ genes showed differential expression, wherein some members were expressed only in the morning or afternoon, in the leaf and/or root tissue, irrespective of the genotypes.

\subsection{AQP gene expression under high VPD conditions}

In terms of relative expression at high VPD (4.21 kPa) conditions when normalized both with corresponding samples under low VPD in the afternoon (1.2 kPa) and reference genes, the expression of PgAQPs in the root tissue of the VPD-insensitive genotype ICMR 1122 was higher than the VPD-sensitive genotype ICMR 1152 and ICMR 1078, where most of the PgAQPs showed lower expression (Fig. 8a). Interestingly, PgPIP2;3 showed the highest expression in root tissue of VPDinsensitive genotypes (ICMR 1122 and ICMR 1086) compared to VPDsensitive ones (ICMR 1152 and ICMR 1078) (Fig. 8a and b). In case of the VPD-sensitive genotypes (ICMR 1152 and ICMR 1078), leaf tissues had higher $P g A Q P$ transcript abundance than in the root tissues with few exceptions (Fig. 8a and b). The expression of PgPIP1;1, PgPIP1;2, PgPIP2;1, PgPIP2;6, PgTIP1;1 and PgTIP2;2 was higher in the leaf tissue (Fig. 8a and b). Especially, in the leaf tissues of the VPD-sensitive genotypes (ICMR 1078), PgTIP2;2 showed higher expression than in the VPD-insensitive genotypes (ICMR 1086) (Fig. 8b). In summary, it can be concluded that PgPIP2;3 showed higher transcript abundance in VPD-insensitive genotypes (ICMR 1122 and ICMR 1086), and PgPIP2;1 showed high transcript abundance in VPD-sensitive genotypes (ICMR 1152 and ICMR 1078), while the rest of the PgAQPs showed differential expression in response to high VPD stress (Fig. 8a and b).

\section{Discussion}

AQPs are transmembrane proteins that play a pivotal role in water transport and also function as gateway for transport of specific substances, including water molecules [20,39]. Plant AQPs comprise of a conserved protein family with diverse members, viz., 35 in A. thaliana, 33 in Z. mays, 33 in O. sativa, 41 in S. bicolor, 41 in S. tuberosum, and 47 in $L$. esculentum $[21,27,32-35]$. Since the genome sequence of pearl millet has not been released yet, a homology-based approach was followed to clone the $A Q P$ genes in this crop, where we have cloned seven $A Q P$ genes, including five PIPs (PgPIP1;1, PgPIP1;2, PgPIP2;1, PgPIP2;3, PgPIP2;6) and two TIPs (PgTIP1;1 and PgTIP2;2) (Table 2). All the cloned PgAQP sequences shared conserved sequences in important domains, i.e., NPA motifs and ar/R residues between PIPs and TIPs of AQPs with other plant species like rice [33] and maize [21] and sorghum [27]. This could suggest that PgAQPs follow a similar physiological mechanism of regulating water transport and plant hydraulics. The predicted subcellular localization of all cloned PgAQPs was specific to plasma membrane. We also observed that the localization of PgTIP1;1 and PgTIP2;2 varies between plasma membrane and vacuole membrane upon using different online tools for prediction. The PgAQPs showed similar localization pattern as that of AQPs from other plant species [28]. Similar results were also reported when $Z$. mays cells were examined for subcellular localization of PIPs by using immunoprecipitation techniques [40]. Further, the deduced protein sequences of the cloned PgAQPs showed high identity with AQPs from $Z$. mays and $O$. sativa as compared to A. thaliana (Fig. 3), thereby suggesting that pearl millet, maize and rice AQP counterparts might have similar biological functions.

A recent study indicates that enhanced water conservation traits/ 

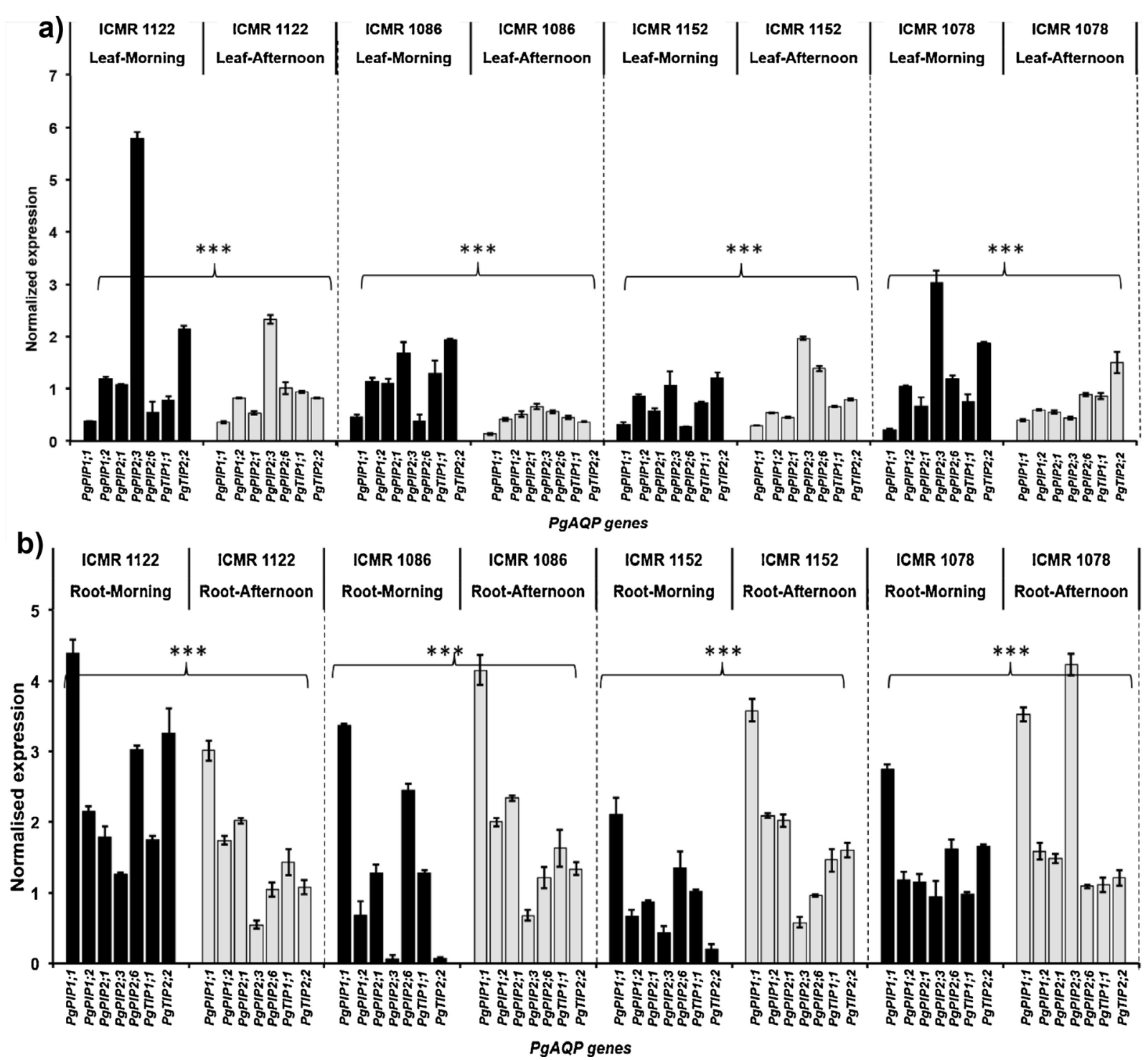

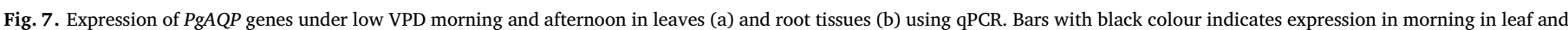

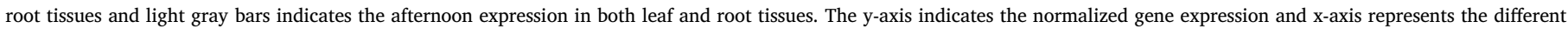

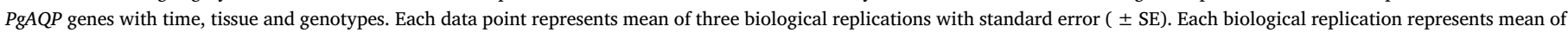

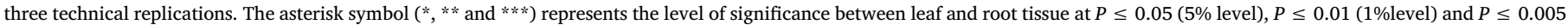
$(0.5 \%$ level). "ns" indicates the non-significant values.

genes would be highly desirable for better drought adaptation in the crops including pearl millet [7], sorghum [41], peanut [16], and cowpea [42] that showed clear genotypic difference at high VPDs. In the present study, transpiration response against the progressive VPD was tested where all the four selected genotypes showed an unrestricted linear transpiration response without breakpoint (Fig. 1). Transpiration restriction under high VPD can also take the form of reduced slopes of response to increasing VPD [11]. In accordance to that, VPD-sensitive genotypes (ICMR 1152 and ICMR 1078) had a lower Tr slope than VPDinsensitive ones (ICMR 1122 and ICMR 1086) under high VPD conditions (Fig. 1). When the gene expression was compared among the tested genotypes, differential $A Q P$ gene expression was observed in roots and leaves of the VPD-insensitive and VPD-sensitive genotypes under low and high VPD conditions (Figs. 4-8). Few of the PgAQPs were expressed only in a specific tissue, time, genotype and VPD conditions. In particular, PgPIP2;3 was highly expressed in leaves during the morning, while in the same genotype, the same AQP was less/downregulated in the afternoon hours in root under low VPD conditions (Figs. 4 and 5). Some PgAQP genes showed either up-regulation during the morning and down-regulation during the afternoon or vice-versa, indicating the likelihood of diurnal expression of AQPs in particular plant organs (Fig. 7). This diurnal expression might be attributed by the localization and varied functions of AQPs during high VPD conditions. Similar results were observed in pea [43], maize [44] and rice [45].

Under high VPD conditions, VPD-insensitive genotypes increased their $\mathrm{Tr}$ as compared to the VPD-sensitive genotypes, may be due to the higher expression of PgAQPs in VPD-insensitive genotypes, compare to VPD-sensitive genotypes (Fig. 8). This increase in Tr might be due to the fact that the VPD-insensitive genotypes utilized more symplastic mediated water transport pathway (AQPs mediated) than the VPDsensitive genotypes (Fig. 8). Though PgPIP2;3 showed higher expression in the roots of VPD-insensitive genotypes (ICMR 1122 and ICMR 1086) and PgPIP2;1 showed higher expression in leaf tissues of VPD-sensitive genotypes (ICMR 1152 and ICMR 1078), other PgAQPs showed clear differential expression (Fig. 8). Our data indicate that PgAQPs from different genotypes respond differently depending upon their tolerance to VPD stress. The differential response of AQP expression to high VPD stress suggests that AQPs belonging to different groups contribute 

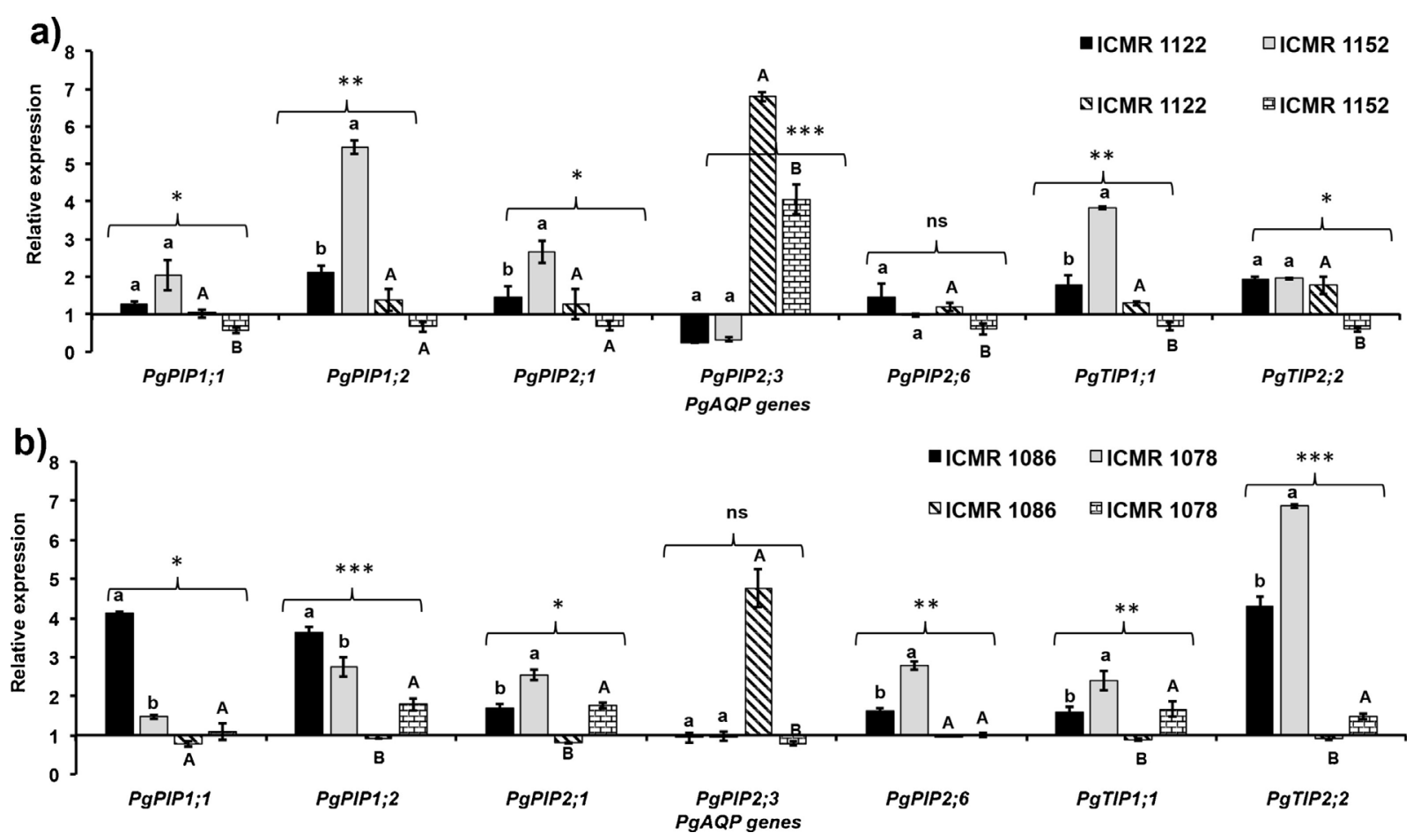

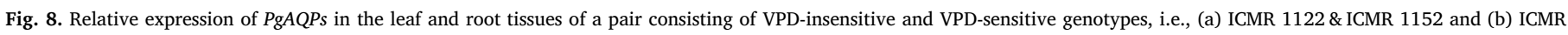

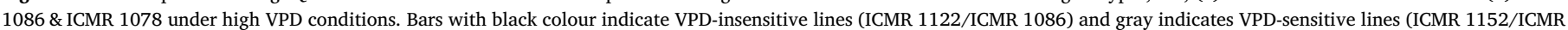

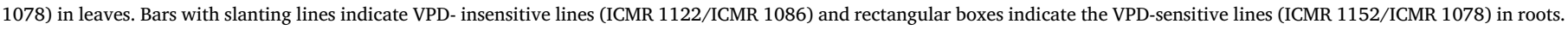

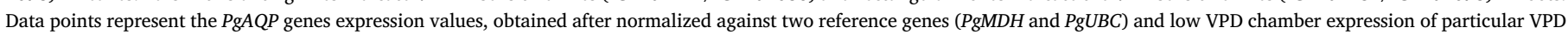

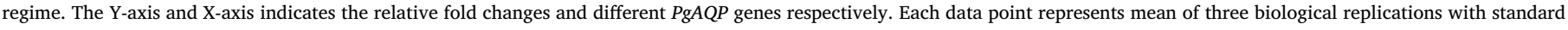

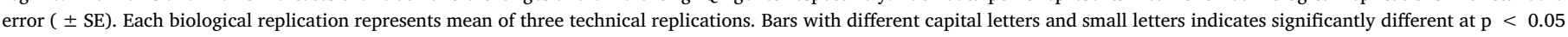

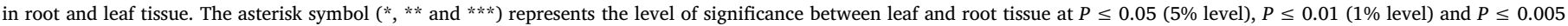
$(0.5 \%$ level). "ns" indicates the non-significant values.

differently to water transport and regulation, influencing the $\operatorname{Tr}(22)$. Preliminary pharmacological approach showed that the Tr of VPD-insensitive genotypes was lowered as compared to that of the VPD-sensitive genotypes when the plants were treated with metal inhibitors. These genotypes might have higher active transcripts of AQPs that are sensitive to the metal inhibitor (Tharanya et al., unpublished data). In summary, it seems that the VPD-insensitive genotypes were able to increase the transcript level of $A Q P$ genes under the challenge of higher VPD conditions, and more so in the roots. These results are also in agreement with recent evidences in soybean [15], peanut [16] and Sorghum [46], wherein no significant decrease in transpiration rate was observed in response to AQPs inhibitor under high VPD conditions. By contrast, VPD-insensitive genotypes had higher inhibition of transcription in response to AQPs inhibitor. This might be due to the contrast in the involvement of AQPs or expression of AQPs under high VPD between VPD-sensitive and -insensitive genotypes. Further studies on elucidation of individual functions of specific $A Q P s$ would lead to better insights on their role in water management in crop plants.

\section{Conclusion}

In this study, we confirmed significant physiological differences in the sensitivity of the transpiration response to increase in VPD condition among inbred lines from a high resolution cross developed to fine map a terminal drought tolerance QTL. The differences in the transpiration response phenotype were accompanied by diverse $A Q P$ gene abundances between the VPD-sensitive and VPD-insensitive genotypes. All the cloned PgAQP genes showed that they were more closely related to maize AQP proteins. While a diurnal rhythm was observed in the expression of the few PgAQP genes, higher expression levels were observed in the morning and a down-regulation in the afternoon and vice versa. Moreover, the expression pattern of the cloned PgAQP genes clearly indicated that the VPD-insensitive genotypes had slightly higher expression of AQPs than in the VPD-sensitive genotypes under high VPD conditions. This supports the hypothesis that some of the AQP proteins could be involved in the regulation and facilitation of water movement under high evaporative demand in pearl millet. On the other hand, the results showing genotypic differences in the level of water uptake demand further detailed study on AQPs.

\section{Author's contribution}

PSR, VV, and KKS conceived the study. PSR, VV and JK designed the experiments. PSR, MT, KS and MS performed the experiments. PSR, KS, CTH and VV interpreted the data. PSR, KKS and VV wrote the paper.

\section{Acknowledgements}

PSR is thankful to the Department of Science and Technology, Govt. of India, New Delhi, for research funding under the Young Scientist Scheme (SB/YS/LS-12/2013) through SERB and INSPIRE Faculty Fellowship Award (Grant No. IFA-LSPA-06). This work has been undertaken as part of the CGIAR Research Program on Dryland Cereals, and received funding from ICRISAT under the Blue Sky Research Project on "Developing crops with high productivity under high temperatures". ICRISAT is a member of the CGIAR Consortium.

\section{Appendix A. Supplementary data}

Supplementary data associated with this article can be found, in the online version, at http://dx.doi.org/10.1016/j.plantsci.2017.10.005. 


\section{References}

[1] H.S. Shetty, S.N. Raj, K.R. Kini, H.R. Bishnoi, R. Sharma, B.S. Rajpurohit, R. Mahala, H.P. Yadav, S.K. Gupta, O.P. Yadav, Downy Mildew of Pearl Millet and Its Management, All India Coordinated Research Project on Pearl Millet (ICAR), Jodhpur, 2016.

[2] S.K. Gupta, K.N. Rai, P. Singh, V.L. Ameta, S.K. Gupta, A.K. Jayalekha, R.S. Mahala, S. Pareek, M.L. Swami, Y.S. Verma, Seed set variability under high temperatures during flowering period in pearl millet (Pennisetum glaucum L. (R.) Br.), Field Crop Res. 171 (2015) 41-53.

[3] V.S. Nambiar, J.J. Dhaduk, N. Sareen, T. Shahu, R. Desai, Potential functional implications of pearl millet (Pennisetum glaucum) in health and disease, J. Appl. Pharm. Sci. 1 (2011) 62-67.

[4] M.E. Gilbert, M.A. Zwieniecki, N.M. Holbrook, Independent variation in photosynthetic capacity and stomatal conductance leads to differences in intrinsic water use efficiency in 11 soybean genotypes before and during mild drought, J. Exp. Bot. 62 (2011) 2875-2887.

[5] A. Nezhadahmadi, Z.H. Prodhan, G. Faruq, Drought tolerance in wheat, Sci. World J. 2013 (2013) 610721.

[6] B.P. Swamy, A. Kumar, Genomics-based precision breeding approaches to improve drought tolerance in rice, Biotechnol. Adv. 31 (2013) 1308-1318.

[7] J. Kholova, C.T. Hash, A. Kakkera, M. Kocova, V. Vadez, Constitutive water-conserving mechanisms are correlated with the terminal drought tolerance of pearl millet [Pennisetum glaucum (L.) R. Br.], J. Exp. Bot. 61 (2010) 369-377.

[8] J. Kholova, C.T. Hash, P.L. Kumar, R.S. Yadav, M. Kocova, V. Vadez, Terminal drought-tolerant pearl millet [Pennisetum glaucum (L.) R. Br.] have high leaf ABA and limit transpiration at high vapour pressure deficit, J. Exp. Bot. 61 (2010) 1431-1440.

[9] J. Kholova, T. Nepolean, C.T. Hash, A. Supriya, V. Rajaram, S. Senthilvel, A. Kakkera, R. Yadav, V. Vadez, Water saving traits co-map with a major terminal drought tolerance quantitative trait locus in pearl millet [Pennisetum glaucum (L.) R. Br.], Mol. Breeding 30 (2012) 1337-1353.

[10] R.S. Yadav, D. Sehgal, V. Vadez, Using genetic mapping and genomics approaches in understanding and improving drought tolerance in pearl millet, J. Exp. Bot. 62 (2011) 397-408.

[11] V. Vadez, J. Kholova, R.S. Yadav, C.T. Hash, Small temporal differences in water uptake among varieties of pearl millet (Pennisetum glaucum (L.) R. Br.) are critical for grain yield under terminal drought, Plant Soil 371 (2013) 447-462.

[12] V. Vadez, J. Kholova, M. Zaman-Allah, N. Belko, Water: the most important 'molecular' component of water stress tolerance research, Funct. Plant Biol. 40 (2013) 1310-1322.

[13] V. Vadez, J. Kholova, S. Medina, A. Kakkera, H. Anderberg, Transpiration efficiency: new insights into an old story, J. Exp. Bot. 65 (2014) 6141-6153.

[14] A.L. Fletcher, T.R. Sinclair, L.H. Allen, Transpiration responses to vapor pressure deficit in well watered 'slow-wilting' and commercial soybean, Environ. Exp. Bot. 61 (2007) 145-151.

[15] W. Sadok, T.R. Sinclair, Transpiration response of 'slow-wilting' and commercial soybean (Glycine $\max (\mathrm{L}$.$) Merr.) genotypes to three aquaporin inhibitors, J. Exp.$ Bot. 61 (2010) 821-829.

[16] M.J. Devi, W. Sadok, T.R. Sinclair, Transpiration response of de-rooted peanut plants to aquaporin inhibitors, Environ. Exp. Bot. 78 (2012) 167-172.

[17] C. Maurel, L. Verdoucq, D.T. Luu, V. Santoni, Plant aquaporins: membrane channels with multiple integrated functions, Annu. Rev. Plant Biol. 59 (2008) 595-624.

[18] K. Prado, C. Maurel, Regulation of leaf hydraulics: from molecular to whole plant levels, Front. Plant Sci. 4 (2013).

[19] F. Chaumont, S.D. Tyerman, Aquaporins: highly regulated channels controlling plant water relations, Plant Physiol. 164 (2014) 1600-1618.

[20] P. Agre, The aquaporin water channels, Proc. Am. Thorac. Soc. 3 (2006) 5-13.

[21] F. Chaumont, F. Barrieu, E. Wojcik, M.J. Chrispeels, R. Jung, Aquaporins constitute a large and highly divergent protein family in maize, Plant Physiol. 125 (2001) 1206-1215.

[22] C. Hachez, F. Chaumont, Aquaporins a family of highly regulated multifunctional channels, Adv. Exp. Med. Biol. 679 (2010) 1-18.

[23] F. Siefritz, B. Otto, G.P. Bienert, A. van der Krol, R. Kaldenhoff, The plasma membrane aquaporin NtAQP1 is a key component of the leaf unfolding mechanism in tobacco, Plant J. 37 (2004) 147-155.

[24] M. Bots, R. Feron, N. Uehlein, K. Weterings, R. Kaldenhoff, T. Mariani, PIP1 and
PIP2 aquaporins are differentially expressed during tobacco anther and stigma development, J. Exp. Bot. 56 (2005) 113-121.

[25] N. Uehlein, R. Kaldenhoff, Aquaporins and plant leaf movements, Ann. Bot.-London 101 (2008) 1-4.

[26] F.W. Ge, P. Tao, Y. Zhang, J.B. Wang, Characterization of AQP gene expressions in Brassica napus during seed germination and in response to abiotic stresses, Biol. Plantarum 58 (2014) 274-282.

[27] P.S. Reddy, T.S.R.B. Rao, K.K. Sharma, V. Vadez, Genome-wide identification and characterization of the aquaporin gene family in Sorghum bicolor (L.), Plant Gene 1 (2015) 18-28.

[28] D.T. Luu, C. Maurel, Aquaporins in a challenging environment: molecular gears for adjusting plant water status, Plant Cell Environ. 28 (2005) 85-96.

[29] Y.H. Peng, W.L. Lin, W.M. Cai, R. Arora, Overexpression of a Panax ginseng tonoplast aquaporin alters salt tolerance, drought tolerance and cold acclimation ability in transgenic Arabidopsis plants, Planta 226 (2007) 729-740.

[30] C. Ehlert, C. Maurel, F. Tardieu, T. Simonneau, Aquaporin-mediated reduction in maize root hydraulic conductivity impacts cell turgor and leaf elongation even without changing transpiration, Plant Physiol. 150 (2009) 1093-1104.

[31] C. Xu, M. Wang, L. Zhou, T. Quan, G. Xia, Heterologous expression of the wheat aquaporin gene TaTIP2;2 compromises the abiotic stress tolerance of Arabidopsis thaliana, PLoS One 8 (2013) e79618.

[32] U. Johanson, M. Karlsson, I. Johansson, S. Gustavsson, S. Sjovall, L. Fraysse, A.R. Weig, P. Kjellbom, The complete set of genes encoding major intrinsic proteins in Arabidopsis provides a framework for a new nomenclature for major intrinsic proteins in plants, Plant Physiol. 126 (2001) 1358-1369.

[33] J. Sakurai, F. Ishikawa, T. Yamaguchi, M. Uemura, M. Maeshima, Identification of 33 rice aquaporin genes and analysis of their expression and function, Plant Cell Physiol. 46 (2005) 1568-1577.

[34] S. Reuscher, M. Akiyama, C. Mori, K. Aoki, D. Shibata, K. Shiratake, Genome-wide identification and expression analysis of aquaporins in tomato, PLoS One 8 (2013).

[35] J. Venkatesh, J.W. Yu, S.W. Park, Genome-wide analysis and expression profiling of the Solanum tuberosum aquaporins, Plant Physiol Bioch 73 (2013) 392-404.

[36] R. Serraj, C.T. Hash, S.M.H. Rizvi, A. Sharma, R.S. Yadav, F.R. Bidinger, Recent advances in marker-assisted selection for drought tolerance in pearl millet, Plant Prod. Sci. 8 (2005) 334-337.

[37] P.S. Reddy, D.S. Reddy, P. Bhatnagar-Mathur, K.K. Sharma, V. Vadez, Cloning and validation of reference genes for normalization of gene expression studies in pearl millet [Pennisetum glaucum (L.) R. Br.] by quantitative real-time PCR, Plant Gene 1 (2015) 35-42.

[38] J. Hellemans, G. Mortier, A. De Paepe, F. Speleman, J. Vandesompele, qBase relative quantification framework and software for management and automated analysis of real-time quantitative PCR data, Genome Biol. 8 (2007).

[39] K.R. Vinothkumar, R. Henderson, Structures of membrane proteins, Q. Rev. Biophys. 43 (2010) 65-158.

[40] E. Zelazny, J.W. Borst, M. Muylaert, H. Batoko, M.A. Hemminga, F. Chaumont, FRET imaging in living maize cells reveals that plasma membrane aquaporins interact to regulate their subcellular localization, Proc. Natl. Acad. Sci. U. S. A. 104 (2007) 12359-12364.

[41] M. Gholipoor, P.V.V. Prasad, R.N. Mutava, T.R. Sinclair, Genetic variability of transpiration response to vapor pressure deficit among sorghum genotypes, Field Crop Res. 119 (2010) 85-90.

[42] N. Belko, M. Zaman-Allah, N. Diop, N. Cisse, G. Zombre, J. Ehlers, V. Vadez, Restriction of transpiration rate under high vapour pressure deficit and non-limiting water conditions is important for terminal drought tolerance in cowpea, Plant Biol. 15 (2013) 304-316.

[43] P.C. Beaudette, M. Chlup, J. Yee, R.J.N. Emery, Relationships of root conductivity and aquaporin gene expression in Pisum sativum: diurnal patterns and the response to $\mathrm{HgCl}_{2}$ and ABA, J. Exp. Bot. 58 (2007) 1291-1300.

[44] D.T. Clarkson, M. Carvajal, T. Henzler, R.N. Waterhouse, A.J. Smyth, D.T. Cooke, E. Steudle, Root hydraulic conductance: diurnal aquaporin expression and the effects of nutrient stress, J. Exp. Bot. 51 (2000) 61-70.

[45] R.M. Nada, G.M. Abogadallah, Aquaporins are major determinants of water use efficiency of rice plants in the field, Plant Sci. 227 (2014) 165-180.

[46] S. Choudhary, T.R. Sinclair, P.V.V. Prasad, Hydraulic conductance of intact plants of two contrasting sorghum lines, SC15 and SC1205, Funct. Plant Biol. 40 (2013) 730-738. 